\title{
When two is too many: Collaborative encoding impairs memory
}

\author{
SARAH J. BARber, Suparna RaJARAM, ANd Arthur Aron \\ Stony Brook University, Stony Brook, New York
}

\begin{abstract}
Humans routinely encode and retrieve experiences in interactive, collaborative contexts. Yet much of what we know about human memory comes from research on individuals working in isolation. Some recent research has examined collaboration during retrieval, but not much is known about how collaboration during encoding affects memory. We examined this issue. Participants created episodes by elaborating on study materials alone or collaboratively, and they later performed a cued-recall task alone, with the study partner, or with a different partner (Experiment 1). Collaborative encoding impaired recall. This counterintuitive outcome was found for both individual and group recall, even when the same partners collaborated across encoding and retrieval. This impairment was significantly reduced, but persisted, when the encoding instructions encouraged free-flowing collaboration (Experiment 2). Thus, the collaborative-encoding deficit is robust in nature and likely occurs because collaborative encoding produces less effective cues for later retrieval.
\end{abstract}

As a social species, we spend the majority of our lives with others, and much of what we later want to remember is encoded in an interactive group context. Even in Western education, in which learning and testing usually involve individual effort, teachers routinely use collaborative learning as a pedagogical tool. Yet we know very little about how collaboration during encoding affects memory. Historically, cognitive research on memory has focused on individuals working in isolation, and group memory has been studied largely within the domains of sociology, social psychology, and anthropology (see, e.g., Echterhoff, Higgins, \& Levine, 2009; Halbwachs, 1950/1980; Hirst \& Manier, 2008; Wegner, 1987; Wertsch, 2002). Recent cognitive research has begun to examine how collaboration affects memory (Barnier \& Sutton, 2008; Harris, Paterson, \& Kemp, 2008; Weldon, 2001); however, this effort has been directed mainly at understanding collaborative retrieval. In the present article we report novel findings on how collaboration during encoding affects memory.

In a typical collaborative-memory paradigm, participants perform the encoding task, such as studying a list of words, individually. At test, participants recall the studied information either alone or in a group. Predictably, groups recall more than any one individual (Yuker, 1955). But to understand how collaboration affects group memory, the comparison is made between the recall of interacting, or collaborative, groups and the recall of nominal groups of equal size. Nominal groups are groups in name only, in which the individual recall of participants is pooled together in a nonredundant fashion, so that overlapping items are counted only once. The outcome of this comparison is counterintuitive: Collaborative groups recall significantly less than do nominal groups, a phenomenon Weldon and Bellinger (1997) termed collaborative inhibition.

Collaborative inhibition during retrieval is a robust finding, occurring routinely in free recall and with a wide variety of study materials (Weldon, 2001). Although social loafing (Karau \& Williams, 1993) may seem an obvious explanation, collaborative inhibition does not occur because of diffusion of responsibility or reduced motivation (Weldon, Blair, \& Huebsch, 2000). Instead, a cognitive explanation, namely retrieval disruption, provides the best explanation (B. H. Basden, D. R. Basden, Bryner, $\&$ Thomas, 1997). Derived from research on the part-list cuing phenomenon in individual memory (D. R. Basden \& B. H. Basden, 1995; Slamecka, 1969), the retrievaldisruption account posits that exposure to already recalled items disrupts an individual's idiosyncratic retrieval strategy for the remaining to-be-recalled items and hence lowers recall. In the collaborative-recall situation, retrieval disruption occurs from hearing others' recalled items, thus lowering each member's (and in turn the group's) recall. This is similar to brainstorming research showing that collaborative groups generate fewer novel ideas than do nominal groups (e.g., Paulus, 2000; see also Nijstad \& Stroebe, 2006).

There is good empirical support for the retrievaldisruption account. For example, collaborative inhibition increases with group size because more members create more disruption during recall (Thorley \& Dewhurst, 2007). Likewise, dyads (the smallest group size) do not reliably produce collaborative inhibition (see, e.g., Meudell, Hitch, \& Kirby, 1992). Also, collaborative inhibition occurs reliably in "uncued" tasks such as free recall 
(in which no test cue is available to aid retrieval) and not in "cued" tasks such as recognition or cued recall, in which retrieval cues are provided (Clark, Hori, Putnam, \& Martin, 2000; Finlay, Hitch, \& Meudell, 2000). This is because idiosyncratic retrieval strategies are important in uncued tasks but not in cued tasks, in which the test cues guide retrieval for both nominal and collaborative groups. In brief, collaboration during an uncued memory test, such as free recall, harms memory.

Collaboration can also affect memory via postevent discussion. In a typical experiment, participants perform the encoding task, such as viewing a series of pictures, individually. Participants then discuss the information (either with another participant or with a confederate) prior to recalling the information individually. In line with expectations, these collaborative discussions provide an opportunity to relearn the study information and this "second study opportunity" often improves later individual performance (see, e.g., Blumen \& Rajaram, 2008; Weldon $\&$ Bellinger, 1997). But there are two additional ways in which collaborative discussion changes later memory. First, the participant's goals during the collaborative discussion will influence not only the amount, accuracy, and type of information that is recalled during the discussion itself (see, e.g., Marsh \& Tversky, 2004; Pasupathi, Lucas, $\&$ Coombs, 2002), but also subsequent memory for the event (see, e.g., Echterhoff, Higgins, \& Groll, 2005; Echterhoff, Higgins, Kopietz, \& Groll, 2008; Higgins \& Rholes, 1978; Pasupathi, Stallworth, \& Murdoch, 1998). For instance, the creative process involved in telling a story for entertainment leads to lasting distortions in a participant's memory for the event compared with telling a story with the goal of accurately conveying the events (Dudukovic, Marsh, \& Tversky, 2004; see also Marsh, 2007). Second, the recollection of the discussion partner can also influence the participant's memory for the event. Misinformation recalled by the discussion partner is often incorporated into the participant's final individual recall (see, e.g., B. H. Basden, Reysen, \& Basden, 2002; French, Garry, \& Mori, 2008; Gabbert, Memon, \& Wright, 2006; Meade \& Roediger, 2002; Roediger, Meade, \& Bergman, 2001), especially when the individual feels pressure to conform to the discussion partner's responses (Reysen, 2007). This is true even when the information has been recalled correctly by the participant earlier in the experiment (Gabbert, Memon, Allan, \& Wright, 2004). Similarly, correct information that the discussion partner does not recall is often omitted from the final individual recall (Merckelbach, Van Roermund, \& Candel, 2007), especially when the discussion partner recalls other related details selectively (Cuc, Koppel, \& Hirst, 2007). Such socially induced forgetting can occur even for information that was held uniquely by an individual if that information did not come up during discussion (Coman, Manier, \& Hirst, 2009). Thus, collaborative discussion in the postencoding phases can change memory.

Although ample research has suggested that collaboration during any postencoding stage can negatively influence retrieval (both on a final critical-memory test and during a discussion), surprisingly little is known about how collaboration during encoding itself affects later memory. This is surprising given the ubiquity of the shared encoding experience and the prevalence of systematically structured collaborative learning in classrooms and study groups. Further, we cannot simply generalize from research on collaborative recall, because research on individual memory often finds that variables produce asymmetrical effects across encoding and retrieval (e.g., Craik, Govoni, Naveh-Benjamin, \& Anderson, 1996; Mulligan \& Lozito, 2006). Therefore, in the present study we asked how collaboration during encoding influences memory.

Educational research examining this issue has focused on collaborative learning, the process by which students work jointly at some educational task (such as encoding information) in small groups. Although most research has shown that collaborative groups outperform individuals during the learning task itself, this benefit does not always carry over to later individual memory performance (Krause, Stark, \& Mandl, 2009; Laughlin \& Sweeney, 1977). During learning, for example, groups consistently created more detailed and higher quality concept maps (a schematic diagram of the interrelationships among concepts and ideas; Kwon \& Cifuentes, 2009) than did individuals. However, across studies the collaborative learning of maps improved, worsened, or left unaffected later knowledge and memory for concepts (Brown, 2003; Kwon \& Cifuentes, 2007, 2009), making it difficult for one to draw clear conclusions from these findings.

Social psychological research examining collaborative learning has proposed that its efficacy may be mediated by the level of transactive memory within the group (Liang, Moreland, \& Argote, 1995). According to the transactive theory of memory, collaborative groups who repeatedly interact develop a system for dividing up the memory labor. Each group member is responsible for the encoding, storing, and retrieving of the information that is related to his or her own area of expertise or group role. Further, group members develop an awareness of how the memory labor is divided. This specialization allows more information to be recalled by distributing some (but not all) of the memory labor nonredundantly across group members (see, e.g., Wegner, 1987; for a review, see Peltokorpi, 2008); thus, groups that have developed a transactive memory system recall more than does any individual (Johansson, Andersson, \& Rönnberg, 2005) or group of strangers (Wegner, Erber, \& Raymond, 1991). Additionally, groups that have developed a transactive memory system are sometimes unaffected by collaborative inhibition. After collaboratively encoding, collaborating older adult couples recall as much as, but not more than, nominal older adult couples (Ross, Spencer, Linardatos, Lam, \& Perunovic, 2004).

Although the transactive-memory literature has shown the efficacy of divided labor and nonredundant distribution of information among close others on later group memory, the present study focuses on a different kind of collaboration that is just as common. In particular, how does memory for episodes that involve shared processing and joint experience compare with memory for individu- 
ally experienced episodes? Furthermore, does memory for such jointly experienced events differ from memory for individually experienced events as a function of whether retrieval occurs alone, with the same partner from encoding, or with a different partner? The present study was designed to address the effects of such collaborative encoding and retrieval conditions on memory outcomes.

We could locate only two studies that directly manipulated collaboration at both encoding and at retrieval. In one (Finlay et al., 2000), collaboration was manipulated both at encoding and at retrieval, but with the purpose of testing the role of retrieval mechanisms. The encoding task was designed to align the sequential organization of stimuli between partners by requiring the dyadic partners to take turns pointing to pictures in a puzzle. This encoding task was well suited for ensuring sequential alignment of the encoded stimuli, since it reduced retrieval disruption; however, it is not as well suited to addressing how joint construction and elaboration of each study episode affects later memory.

A second study (Andersson \& Rönnberg, 1995) tested the effects of several factors on collaborative memory, including individual versus collaborative encoding. Retrieval involved two consecutive recall tasks in which the first recall was always individual and the second was either individual or collaborative. Individual recall was not affected by the encoding condition on either the first or second recall, but, somewhat counterintuitively, collaborative recall was impaired after collaborative encoding on the second recall (when compared with the nominal recall that was derived from the first, individual, recall scores). The complex design and procedure of this experiment make it difficult to pinpoint exactly why collaborative encoding produced these differential effects across individual retrieval and collaborative retrieval. Furthermore, for collaborative encoding, participants viewed a 34-min videotape individually and discussed it as dyads. But a 2-min break that occurred between the viewing and the discussion likely exceeded the duration of working memory (Baddeley, 1986; Gathercole, 2008) and turned this collaborative discussion into a retrieval task. If this discussion served as a functional collaborative recall, then, as previously shown, it may have exerted positive effects on later individual recall through re-exposure effects (Blumen \& Rajaram, 2008; Rajaram \& Pereira-Pasarin, 2007; Weldon \& Bellinger, 1997). At the same time, as the authors hypothesized, this discussion presumably reduced the specificity of the cues generated at encoding and thus exerted negative effects on the second, collaborative recall (Mäntylä \& Nilsson, 1983). In brief, these differential effects of collaborative encoding are intriguing and open new questions about how joint encoding affects later individual and collaborative recall.

Taken together, the scant evidence on shared encoding raises tantalizing questions about how collaborative elaboration affects encoding and recall, and it motivates the need for targeted investigation; therefore, the present study directly tested two critical issues: (1) Does joint construction and elaboration of study episodes affect the encoding process and later recall? (2) Does this collaborative-encoding process differentially affect later recall depending on whether recall occurs alone, with the same partner from encoding, or with a different partner? To answer these questions, we used an encoding task (adapted from Graf \& Schacter, 1985) that required participants to construct unique study episodes. Participants were presented unrelated word pairs and instructed to create, individually or dyadically, a sentence linking the words in a meaningful way. In this task, the constructed sentences were unique from one word pair to the next, and unique for the same word pair from one participant (or dyad) to the next. Further, a sentence construction task engenders deep and meaningful processing of word pairs and promotes memory for new associations (Graf $\&$ Schacter, 1985). As described below, we evaluated the quality of the generated sentences.

In keeping with our focus on the encoding process and on the interaction between encoding and retrieval, conditions were designed to minimize collaborative inhibition resulting from retrieval processes. Specifically, we used dyads (at both study and test) and a cued-recall task at test, conditions under which collaborative inhibition dissipates (B. H. Basden, D. R. Basden, \& Henry, 2000; Finlay et al., 2000).

The experiment included five between-subjects conditions. Participants (1) encoded individually and recalled individually, (2) encoded individually and recalled dyadically, (3) encoded dyadically and recalled individually, (4) encoded dyadically and recalled dyadically with the same partner, or (5) encoded dyadically and recalled dyadically with a different partner. The most straightforward predictions arise from the encoding-specificity hypothesis, which posits that recall should be better when conditions at retrieval match conditions at encoding than when they mismatch (Tulving, 1983; Tulving \& Osler, 1968). Given that memories are associated with a wide variety of contextual cues at encoding (such as the place of learning, the mood of the participant, etc.; for a review, see Rajaram \& Barber, 2008), recall is enhanced when the cues from encoding are available at retrieval. Collaboratively created memories should thus be recalled better when recalling with the same partner versus when recalling individually or recalling dyadically with a new partner. Similarly, individually created memories should be recalled better when recalling individually than when recalling dyadically. Note that this predicted pattern (lower memory in a collaborative setting than in an individual setting after individual encoding) is identical to the collaborative-inhibition effect that occurs as a result of retrieval factors. Our use of dyads and the cued-recall task minimized the role that retrieval processes could play here in reducing recall, however. As such, if dyadic recall is lower in this condition, it would likely be due to the benefits of the study-test match posited by the encodingspecificity hypothesis.

However, alternate, and perhaps less intuitive, predictions arise from research on the role of cue uniqueness in recall that documents superior recall when the cues given at recall are self-generated rather than generated by others (Mäntylä \& Nilsson, 1983) or dyadically gen- 
erated (Andersson \& Rönnberg, 1997). Consequently, in the present study, recall should be higher following individual encoding than following collaborative encoding, regardless of whether the recall is individual or collaborative. We tested these competing predictions in Experiment 1.

\section{EXPERIMENT 1}

\section{Method}

\section{Participants and Design}

Two hundred forty college volunteers participated for course credit, with 48 participants ( 24 dyads, always strangers) assigned to each condition: (1) encode alone-recall alone, (2) encode alonerecall dyadically, (3) encode dyadically-recall alone, (4) encode dyadically-recall dyadically with the same partner from encoding, and (5) encode dyadically-recall dyadically with a different partner from encoding (two dyads simultaneously completed the encoding and were crossed as partners during retrieval).

\section{Materials}

We generated 130 unrelated nouns from the MRC Psycholinguistic Database (Wilson, 1988), each with a unique first trigram, 4-7 letters in length, and 30-100 per million in word frequency, and we combined them into 65 unrelated paired associates.

\section{Procedure}

Encoding. During the first part of the experiment, participants completed an incidental encoding task, which was described to them as a sentence-creation task. During this task, participants saw pairs of words, such as citizen-trail, and created sentences using these words such that the first word of the pair (e.g., citizen) came earlier in the sentences than did the second word of the pair (e.g., trail). In the encode-alone conditions, participants worked alone to create a sentence from each word pair (e.g., for the pair citizen-trail, "The citizen went along the trail."). In the encode dyadically conditions, partners were instructed to alternate beginning and finishing the sentences across the word pairs to ensure equivalent participation; thus, the first participant might have begun the sentence with "The citizen went," and the second participant might have completed the sentence with "along the trail." Participants in the encode dyadically conditions were further instructed not to carry on conversation during this task and to speak only when adding their portion of the sentences. In all condi- tions, participants were shown one word pair at a time (in the same order for all participants) and created the sentences aloud, and the experimenter recorded them. No time limit was imposed during the encoding task; however, we did record the length of time spent. The encoding task took $23.6 \mathrm{~min}(S D=5.37)$ on average. Dyads took significantly longer (24.48 $\mathrm{min})$ than did individuals $(22.81 \mathrm{~min})$ $\left[t(163)=-2.23, M S_{\mathrm{e}}=4.91, p=.03, d=-0.35\right]$.

Filled delay. All participants completed numeric tasks individually for $45 \mathrm{~min}$ in order to prevent ceiling effects on the subsequent memory test.

Retrieval. After the delay, participants completed a surprise cued-recall test. During this test, participants viewed the first word of every pair (e.g., citizen), presented in a random order that was different from the order at encoding, and attempted to recall the corresponding target (e.g., trail). Participants were required to complete this test in a set order, so that they could not return to a previous cue item to change their response after they had moved on to the next cue item. Dyads generated one answer per cue and resolved all disputes internally. No time limit was imposed during this task, and the cued-recall task took $14.5 \mathrm{~min}(S D=4.53)$ on average. Dyads took significantly longer (17.92 $\mathrm{min})$ than did individuals (13.86 $\mathrm{min})$ $\left[t(163)=-2.49, M S_{\mathrm{e}}=3.77, p=.01, d=-0.39\right]$.

\section{Results}

Recall of collaborative dyads was compared with recall of nominal dyads (the pooled, nonredundant answers of two individuals). Where relevant, recall of single individuals is also reported. Sentence cohesiveness was assessed to determine how collaboration affected the encoding process. Alpha level for all analyses in this and the next experiment was set at .05 unless reported otherwise.

We first analyzed correct group recall. For nominal dyads, we defined correct answers as those occurring either when both individuals correctly recalled the target, or when only one individual correctly recalled the target (regardless of how the other individual responded). Correct group recall (collaborative or nominal) differed significantly among the five conditions $[F(4,115)=7.37$, $\left.M S_{\mathrm{e}}=.02, \eta_{\mathrm{p}}^{2}=.20\right]$ (Figure 1). We next tested the competing predictions that were derived from the encodingspecificity hypothesis on the one hand, and from the cueuniqueness hypothesis on the other.

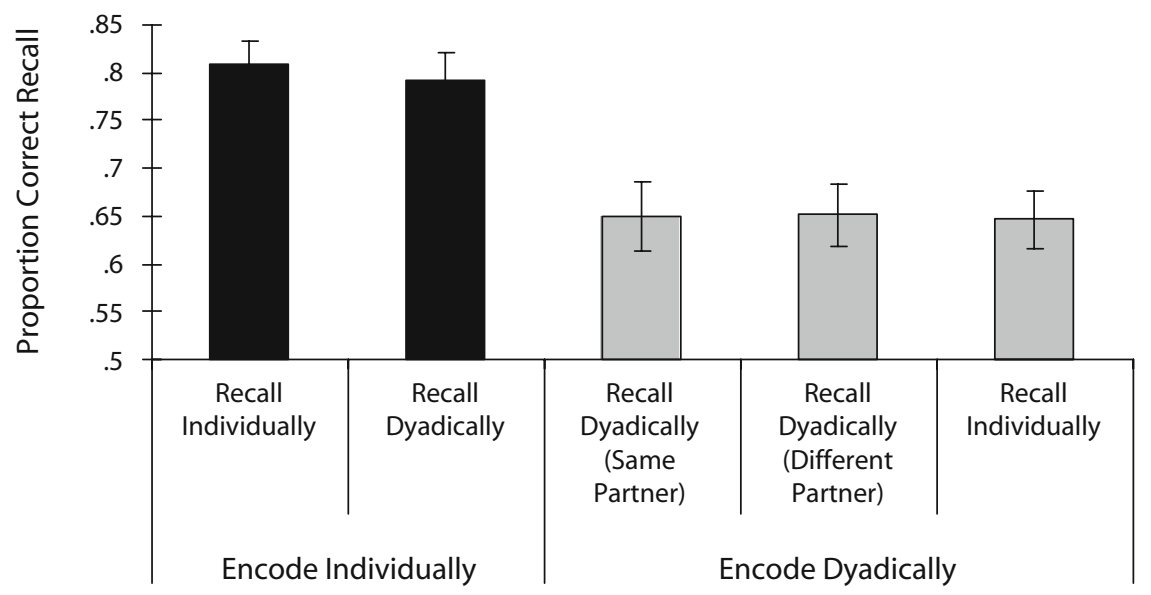

Figure 1. Mean proportion of items correctly recalled by collaborative and nominal groups as a function of encoding and retrieval conditions in Experiment 1. (Error bars are $\pm 1 S E$.) 


\section{Encoding-Specificity Hypothesis}

Contrary to expectations, after dyadic encoding, there was no benefit from recalling dyadically with the same partner (.65) compared with recalling alone or with a different partner (both .65) (both $t \mathrm{~s}<1$ ). Similarly (and again contrary to the predictions), after individual encoding, there was no benefit from recalling individually $(.81)$ compared with recalling dyadically $(.79)(t<1)$.

In fact, only one comparison yielded some support for encoding specificity: Nominal recall was significantly higher in the encode alone-recall alone (encoding-retrieval match) condition (.81) than in the encode dyadicallyrecall alone (mismatch) condition $(.65)[t(115)=3.77$, $\left.M S_{\mathrm{e}}=.04, d=0.70\right]$. But the influence of this pattern was negated by the absence of a match advantage in the encode dyadically-recall dyadically (match) condition (.65) over the encode alone-recall dyadically (mismatch) condition (.79) $\left[t(115)=3.68, M S_{\mathrm{e}}=.04, d=0.61\right]$. Taken together, the observed patterns of recall failed to support the encoding-specificity hypothesis.

\section{Cue-Uniqueness Hypothesis}

The cue-uniqueness hypothesis predicts higher recall following individual encoding than following dyadic encoding. As predicted, a planned comparison showed that group recall (nominal or collaborative) was significantly higher after encoding alone than after encoding dyadically $\left[t(115)=5.4, M S_{\mathrm{e}}=0.17, d=1.01\right]$. This result was replicated for individual (instead of nominal group) memory performance as well in the conditions in which participants retrieved alone. Participants in the encode alone-recall alone condition had significantly higher individual recall (.58) than did participants in the encode dyadically-recall alone condition $(.43)[F(1,94)=14.76$, $\left.M S_{\mathrm{e}}=.038, \eta_{\mathrm{p}}^{2}=.14\right]$. Thus, these results demonstrate a collaborative-encoding deficit such that collaborative encoding impairs memory compared with individual encoding, and the cue-uniqueness hypothesis provides the most parsimonious explanation for this surprising finding.

\section{Errors}

We also analyzed errors on the cued-recall test. We defined errors both as new intrusions and as items paired with the incorrect cue word (e.g., incorrectly recalling the word trail as being paired with animal rather than with citizen). Furthermore, for nominal dyads we defined errors as occurring either when both individuals made an error or when one individual made an error and the other individual did not respond; however, we did not consider the nominal group to have made an error if one individual responded correctly, even if the other individual responded incorrectly.

As in previous studies (Ross, Spencer, Blatz, \& Restorick, 2008), errors in collaborative recall were significantly lower than in nominal recall $\left[t(115)=2.64, M S_{\mathrm{e}}=\right.$ $.05, d=0.49]$. However, errors did not vary between collaborative and individual encoding (encode alone-recall alone, .07; encode alone-recall dyadically, .04; encode dyadically-recall alone, .06; encode dyadically-recall dyadically with the same partner from encoding, .05; and en- code dyadically-recall dyadically with a different partner from encoding, .08) $(t<1)$. Therefore, although collaborative encoding has a negative impact on veridical memory, it does not seem to have an impact on error rates.

\section{Cohesiveness Ratings of Sentences Constructed at Encoding}

Two raters, blind to conditions, independently coded each sentence for cohesiveness on a 4-point scale. Sentences considered "completely cohesive" (rated 4) had well-integrated ideas and clauses (e.g., "White cloth is used to surrender in a battle"). Sentences considered "not at all cohesive" (rated 1) were typically two clauses with little connection between them (e.g., "I have some cloth, but every day is a battle"). The raters showed excellent agreement on the 10,920 sentences (complete agreement on $73.2 \%$ and close agreement [1-point difference] on an additional $12.3 \%$ sentences). Because Rater A used the full range of the scale more often than did Rater B, her scores were used in subsequent analyses.

The average cohesiveness ratings by nominal or collaborative dyads were significantly different across conditions $\left[F(3,92)=22.82, M S_{\mathrm{e}}=.05, \eta_{\mathrm{p}}^{2}=.43\right]^{1}$ (Figure 2), so that individually created sentences were significantly more cohesive than collaboratively created sentences $[t(92)=$ $\left.8.23, M S_{\mathrm{e}}=.18, d=1.72\right]$. Further, a median-split analysis showed that participants who created more cohesive sentences had significantly higher recall (.77) than did those who created less cohesive sentences $(.68)[F(1,94)=$ $\left.9.68, M S_{\mathrm{e}}=.024, \eta_{\mathrm{p}}^{2}=.09\right]$. Similarly, sentences rated as cohesive were recalled marginally better (.55) than were sentences rated as not cohesive $(.52)[F(1,7141)=2.87$, $\left.M S_{\mathrm{e}}=.25, p=.09, \eta_{\mathrm{p}}^{2}<.01\right]$. This was due largely to those in the individual encoding condition having both more cohesive sentences and better recall.

Collaborative encoding thus produced counterintuitive effects on memory: It led to less cohesive encoding and impaired memory on all three recall measuresindividual, nominal, and collaborative. These pervasive impairments run counter to the general intuition about memory for shared encoding. Interestingly, the finding of impaired individual recall is also asymmetrical to the positive cascading effects that are seen when individual recall is preceded by collaborative recall (instead of by collaborative encoding). Collaborative recall benefits later individual memory, because individuals are re-exposed to study stimuli in a collaborative-recall environment (see, e.g., Weldon \& Bellinger, 1997); however, the present findings suggest that collaborative encoding impairs later individual (and collaborative) memory because of suboptimal cues created at encoding.

Social loafing (Karau \& Williams, 1993) cannot readily account for the present findings, since we required participation by each partner during collaborative encoding. Further, we also examined individual recall following dyadic encoding. During dyadic encoding, participants were either the one to start the sentence using the first word (e.g., "The citizen went"), or the one to end the sentence using the second word (e.g., "along the trail"). Individual recall (following collaborative encoding) was equivalent for items 


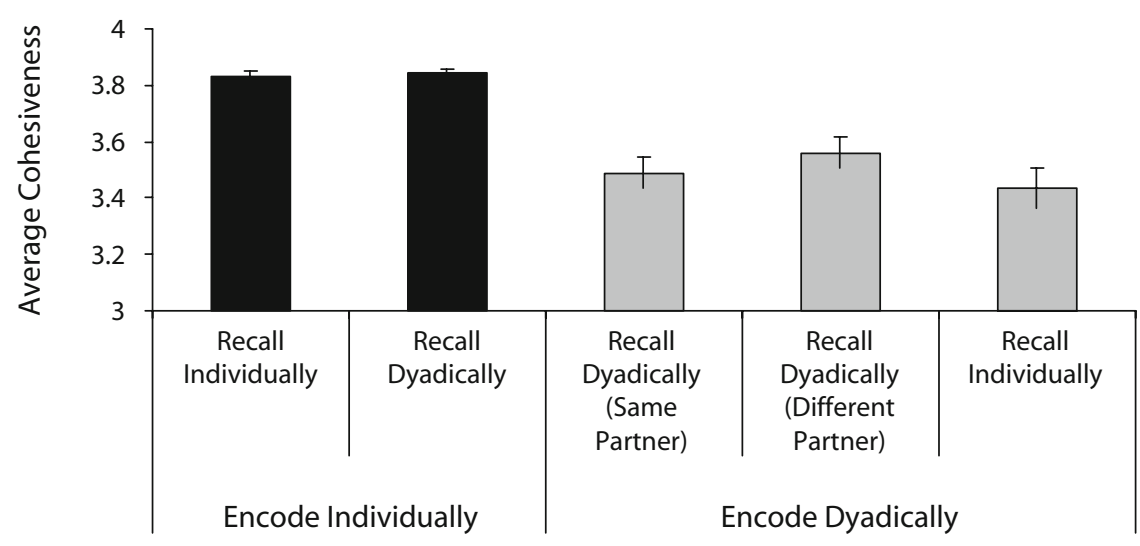

\section{Condition}

Figure 2. Mean cohesiveness of sentences created at encoding by collaborative and nominal groups as a function of encoding and retrieval conditions in Experiment 1. (Error bars are $\pm 1 S E$.

whether the participant started (.44) or completed (.42) the sentences $(F<1)$, showing that partners remained engaged in the encoding task whether or not it was their turn.

In addition, the collaborative-encoding deficit is not simply caused by collaborative-encoding partners having a greater overlap, or redundancy, in their memories. Collaborative encoding lowered not only nominal-group recall but also individual recall. As a further test of redundancy, we analyzed the proportion of items correctly recalled by both individuals of the pair in nominal groups. A greater proportion of items were recalled correctly by both individuals in the nominal pairs after individual encoding (.21) than after dyadic encoding (.35) $\left[t(46)=-2.99, M S_{\mathrm{e}}=.05, d=\right.$ $-0.86]$, further suggesting that the collaborative-encoding deficit is not simply the result of a redundancy in recall across the dyadic encoding partners. In other words, collaborative encoding leads to less information being recalled by each individual within the nominal pair rather than to the same information being recalled by each individual. The specifics of our encoding task suggest a different possibility: Enforced collaboration through turn-taking may curtail the natural, free-flowing process of collaboration that occurs in most human informal social interaction and therefore produces ineffective retrieval cues. If this is the case, allowing dyads to engage in free-for-all collaborative encoding (e.g., without imposing any rules) should improve recall compared with turn-taking.

We tested this possibility by including two conditions from Experiment 1-encode alone and encode dyadically with turn-taking - and adding a third, new conditionencode dyadically via a free-for-all method. Recall in all conditions was performed individually, since the goal was to assess the effects of different methods of collaborative encoding. We expected to replicate the finding from Experiment 1 that turn-taking collaborative encoding produces less effective cues and lower recall than does individual encoding. If free-for-all collaboration facilitates encoding, then we expected this method to produce superior recall compared with the turn-taking method.

\section{EXPERIMENT 2}

\section{Method}

\section{Participants and Design}

Seventy-two college volunteers participated for course credit, with 24 participants (12 dyads, always strangers) in each condition: (1) encode alone, (2) encode dyadically via a turn-taking method, and (3) encode dyadically via a free-for-all method. Cued recall was always performed individually.

\section{Materials and Procedure}

The materials and the procedure for the replication conditions were the same as in Experiment 1. Participants in the new (encode dyadically via a free-for-all method) condition collaborated to create sentences without any specific rules save for the requirement to jointly create a single sentence for each word pair using any method that they chose. Encoding times significantly differed across conditions (encode alone, $26.96 \mathrm{~min}$; turn-taking, $28.83 \mathrm{~min}$; free-for-all, $34.16 \mathrm{~min})\left[F(2,45)=3.54, M S_{\mathrm{e}}=59.07, \eta_{\mathrm{p}}^{2}=.14\right]$, and retrieval times marginally differed across conditions (encode alone, $12.33 \mathrm{~min}$; turn-taking, $14.46 \mathrm{~min}$; free-for-all, $12.04 \mathrm{~min}$ ) $\left[F(2,69)=2.79, M S_{\mathrm{e}}=14.99, p=.07, \eta_{\mathrm{p}}^{2}=.08\right]$.

\section{Results}

Since all conditions entailed recalling alone, individual recall data are reported. Nominal-group scores yielded a similar pattern of findings for all measures. Sentence cohesiveness was again assessed to examine how collaboration affected cue effectiveness.

There were significant differences in correct recall among the conditions $\left[F(2,69)=9.31, M S_{\mathrm{e}}=.04, \eta_{\mathrm{p}}^{2}=\right.$ .21] (Figure 3). Interestingly, free-for-all collaborative encoding significantly increased subsequent individual recall compared with turn-taking collaborative encoding $\left[t(69)=-2.21, M S_{\mathrm{e}}=.06, d=-0.53\right]$; however, recall remained significantly lower after free-for-all collaborative encoding (.63) than after individual encoding (.75) $\left[t(69)=2.10, M S_{\mathrm{e}}=.06, d=0.51\right]$, and significantly lower after turn-taking collaborative encoding (.50) than after individual encoding $\left[t(69)=4.32, M S_{\mathrm{e}}=.06, d=\right.$ 1.04]. The pattern of recall errors was consistent with the 


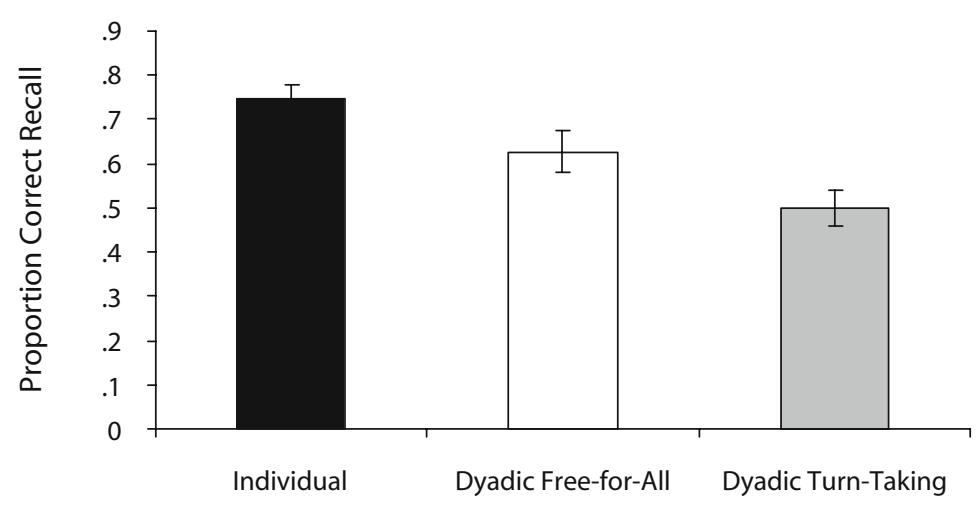

Encoding Condition

Figure 3. Mean proportion of items correctly recalled by individuals as a function of encoding condition in Experiment 2. (Error bars are $\pm 1 S E$.)

accuracy data (free-for-all, .09; turn-taking, .10; encode alone, .07); thus, collaborative encoding produced recall impairment regardless of the collaboration method that was used.

\section{Cohesiveness Ratings of Sentences Constructed at Encoding}

Two raters (including Rater A from Experiment 1) independently rated sentence cohesiveness while blind to experimental conditions. The raters showed excellent interrater agreement on the 6,240 sentences (complete agreement on $76.3 \%$ and close agreement [1-point difference] on an additional $22.1 \%$ sentences.) Rater A's ratings were used in subsequent analyses.

The average cohesiveness ratings differed significantly among the conditions $\left[F(2,69)=49.54, M S_{\mathrm{e}}=.05, \eta_{\mathrm{p}}^{2}=\right.$ .59]. Individual encoding led to significantly more cohesive sentences (3.80) than did turn-taking collaborative encoding (3.24) $\left[t(69)=8.84, M S_{\mathrm{e}}=.06, d=2.13\right]$ but, interestingly, did not differ significantly from freefor-all collaborative encoding (3.77) $(t<1)$. As before, a median-split analysis showed that recall was significantly higher for participants who created cohesive sentences at encoding (.72) than for those who created less cohesive sentences $(.54)\left[F(1,70)=13.56, M S_{\mathrm{e}}=.04, \eta_{\mathrm{p}}^{2}=.16\right]$. Similarly, sentences rated as cohesive were recalled better (.64) than were sentences rated as not cohesive (.45) $\left[F(1,4678)=59.40, M S_{\mathrm{e}}=.23, \eta_{\mathrm{p}}^{2}=.01\right]$. Again, this effect was due largely to more cohesive sentences and better recall for participants in the individual (rather than dyadic) encoding condition.

\section{Encoding Strategies in Free-for-All Collaborative Encoding}

In the free-for-all encoding condition, dyads were at liberty to use any strategy they wanted for jointly creating sentences. This enabled a finer-grained analysis of the performance to see how distinct patterns of collaboration during encoding can influence the quality of the generated cues and subsequent recall.
Of the 12 dyads, 8 chose to primarily use an alternating whole sentences strategy in which one person in the dyad created a sentence, and then the other person in the dyad created the next sentence. The other 4 dyads chose to primarily use a turn-taking within a sentence strategy, with each partner providing pieces to construct the sentence, as was done in Experiment 1. Recall differences between these two subgroups were in the direction that was predicted by the main findings of our experiments; the alternating whole sentences strategy subgroup (whose approach was closer to individual encoding) showed significantly higher individual recall (.69) than did the turn-taking within a sentence strategy subgroup (.51) $\left[F(1,22)=3.90, M S_{\mathrm{e}}=.05, p=.03\right.$ (one-tailed), $\eta_{\mathrm{p}}^{2}=$ .15]. In fact, recall of the alternating whole sentences strategy subgroup was equivalent to the encode-alone condition $(.75)[t(38)=1.02, p=.32]$. Similarly, recall of the turn-taking within a sentence strategy subgroup (of the free-for-all encoding condition) was almost identical numerically to the turn-taking encoding condition in Experiment $2(.50)$, and these two groups did not differ significantly $[t(30)<1]$.

A converging pattern emerged from the analysis of construction strategy at the sentence level. Although all dyads had a dominant strategy, they occasionally used other methods as well. For example, a dyad might have largely used a turn-taking within a sentence strategy (say, on 59 sentences) but used the alternating whole sentences strategy on 6 sentences. A correlational analysis between the number of sentences that participants constructed using the turn-taking within a sentence method and individual recall scores was negative and significant $(r=$ -.47). Similarly, as the number of turn-taking sentences increased, the average cohesiveness of the dyad's sentences significantly decreased $(r=-.87)$. This suggests that a turn-taking strategy that requires equivalent input on each item is associated with both poorer cohesiveness and poorer recall.

We next examined whether participants who used an alternating whole sentences strategy preferentially re- 
membered the sentences that they had created (in entirety) over sentences that their partners had created (in entirety). As expected, participants recalled more of the sentences they had individually created (.71) than sentences that their partners had created (.64), although this numerical pattern failed to reach statistical significance $[t(15)=1.48, p=.08$, one-tailed]. Furthermore, recall of the individually created sentences was equivalent to recall in the encode-alone condition $(.75)(t<1)$, but recall of the partner's created sentences was significantly worse than recall in the encode-alone condition $[t(40)=$ $2.04, p=.05]$. Thus, the attenuation of the collaborativeencoding deficit in the dyadic free-for-all condition may be driven by the memory advantage that is associated with individual encoding.

Recall for sentences that the partner created was still inferior to recall after individual encoding. This outcome may seem counterintuitive, because the sentences in the dyadic free-for-all condition were as cohesive (and thus presumably as memorable) as sentences in the encodealone condition. But cues generated by others, even if qualitatively good, are less effective for later recall than are self-generated cues (Mäntylä \& Nilsson, 1983); thus, collaborative encoding via an alternating whole sentences strategy results in fewer sentences generated by the self, and thus fewer items recalled (numerically, compared with individual encoding), but it still fares better than a turntaking within a sentence strategy. This again underscores the detrimental effects of collaborative encoding.

\section{DISCUSSION}

The findings reported here document that collaborative encoding produces pervasive decrements in recall. Compared to individual encoding, collaborative encoding lowered all measures of recall - individual, nominal, and collaborative. This result held true even when the same dyads collaborated at study and at test. The regularity of this collaborative-encoding impairment is even more striking given that the recall conditions were designed to minimize retrieval-based collaborative deficits. The locus of the observed collaborative-encoding deficit was therefore mainly in the encoding stage, and the deficit was distinct from the previously reported collaborative-inhibition effect that occurs during recall.

The collaborative-encoding deficit occurred regardless of whether the encoding task required partners to contribute equally to the sentence-construction task (Experiment 1) or to use a free-flowing method that imposed no rules for how to collaborate (Experiment 2). If fact, the only time that a collaborative-encoding condition was equivalent to individual encoding (the alternating whole sentences strategy subgroup in Experiment 2), the result turned out to be driven by a memory advantage for individually created encoding episodes, and especially so when the entire sentence was created by oneself rather than one's partner. Therefore, as with the collaborativeinhibition effect at recall, the collaborative-encoding impairment effect is robust in nature.
The negative effects of collaboration at encoding may be due to a deficit in the efficacy of collaboratively created retrieval cues. At encoding, the quality of sentences generated collaboratively (when participants contributed equally in creating each sentence) was qualitatively worse than the quality of sentences generated individually. In particular, collaborating dyads linked items in a less cohesive fashion than did individuals working alone. This may have been especially problematic given that participants were later tested with a cued-recall test, which depends not only on memory for the items, but also on memory for their associations. Even when the quality of the sentences generated collaboratively was qualitatively good (when participants alternated creating whole sentences in Experiment 2), however, the collaborative-encoding deficit persisted. This is in line with previous work demonstrating that self-generated cues are superior to other-generated cues in guiding recall, even when the cues do not qualitatively differ (Mäntylä \& Nilsson, 1983).

The present study documents the nature and effects of collaborative encoding under the most basic conditions necessary to establish the core phenomenon. Nonetheless, the present findings raise questions about the real-world implications of collaboration in terms of how it affects educational practices, eyewitness accounts, and social interactions, and how its cognitive costs might be balanced against its adaptive advantages. Further, the extent to which collaborative encoding could harm or benefit memory would also depend on a myriad of other factors that have long been considered important for shaping individual memory (Jenkins, 1979), and by a host of additional factors related to the social context, such as the nature of the relationship (Hinsz, Tindale, \& Vollrath, 1997). In educational settings, for instance, collaborative learning often involves more complex tasks than the ones employed in the present experiments. Students are often asked to jointly analyze complex problems or case studies, generate applications to concepts, debate opposing views, and respond critically to one another's work. Presumably these activities engage students and induce them to synthesize and organize information effectively, possibly leading to a collaborative-encoding benefit. Although the collaborative-encoding findings reported here document a collaborative-encoding deficit, it remains open to future research to explore these additional factors in order to determine where collaboration may help, hurt, or have no effect on the encoding process across a wider variety of situations.

\section{AUTHOR NOTE}

Special thanks to Rose Slupski, and to Danielle DellaRagione, Yvonne Gao, and Suzanne Riela for research assistance. Correspondence concerning this article should be addressed to S. Rajaram, Department of Psychology, Stony Brook University, Stony Brook, NY 11794-2500 (e-mail: suparna.rajaram@sunysb.edu).

\section{REFERENCES}

Andersson, J., \& RönNBERG, J. (1995). Recall suffers from collaboration: Joint recall effects of friendship and task complexity. Applied Cognitive Psychology, 9, 199-211. doi:10.1002/acp.2350090303 
ANDERsson, J., \& RönNBERG, J. (1997). Cued memory collaboration: Effects of friendship and type of retrieval cue. European Journal of Cognitive Psychology, 9, 273-287. doi:10.1080/713752558

BAdDEley, A. [D.] (1986). Working memory. Oxford: Oxford University Press, Clarendon Press.

Barnier, A. J., \& Sutton, J. (2008). From individual to collective memory: Theoretical and empirical perspectives. Memory, 16, 177182. doi: $10.1080 / 09541440701828274$

BASDEN, B. H., BASDEN, D. R., BRYNer, S., \& ThOMAS, R. L., III (1997). A comparison of group and individual remembering: Does collaboration disrupt retrieval strategies? Journal of Experimental Psychology: Learning, Memory, \& Cognition, 23, 1176-1189. doi:10.1037/0278 -7393.23.5.1176

Basden, B. H., Basden, D. R., \& Henry, S. (2000). Costs and benefits of collaborative remembering. Applied Cognitive Psychology, 14, 497-507. doi:10.1002/1099-0720(200011/12)14:6<497::AID -ACP665>3.0.CO;2-4

Basden, B. H., Reysen, M. B., \& Basden, D. R. (2002). Transmitting false memories in social groups. American Journal of Psychology, 115, 211-231. doi:10.2307/1423436

BAsden, D. R., \& BASDEN, B. H. (1995). Some tests of the strategy disruption interpretation of part-list cuing inhibition. Journal of Experimental Psychology: Learning, Memory, \& Cognition, 21, 1656-1669. doi:10.1037/0278-7393.21.6.1656

BLumen, H. M., \& RAJARAM, S. (2008). Influence of re-exposure and retrieval disruption during group collaboration on later individual recall. Memory, 16, 231-244. doi:10.1080/09658210701804495

BRown, D. S. (2003). High school biology: A group approach to concept mapping. American Biology Teacher, 65, 192-197.

Clark, S. E., Hori, A., Putnam, A., \& Martin, T. P. (2000). Group collaboration in recognition memory. Journal of Experimental Psychology: Learning, Memory, \& Cognition, 26, 1578-1588. doi:10.1037/0278-7393.26.6.1578

Coman, A., Manier, D., \& Hirst, W. (2009). Forgetting the unforgettable through conversation: Socially shared retrieval-induced forgetting of September 11 memories. Psychological Science, 20, 627-633. doi:10.1111/j.1467-9280.2009.02343.x

Craik, F. I. M., Govoni, R., Naveh-Benjamin, M., \& Anderson, N. D. (1996). The effects of divided attention on encoding and retrieval processes in human memory. Journal of Experimental Psychology: General, 125, 159-180. doi:10.1037/0096-3445.125.2.159

Cuc, A., Koppel, J., \& Hirst, W. (2007). Silence is not golden: A case for socially shared retrieval-induced forgetting. Psychological Science, 18, 727-733. doi:10.1111/j.1467-9280.2007.01967.x

Dudukovic, N. M., Marsh, E. J., \& Tversky, B. (2004). Telling a story or telling it straight: The effects of entertaining versus accurate retellings on memory. Applied Cognitive Psychology, 18, 125-143. doi:10.1002/acp.953

Echterhoff, G., Higgins, E. T., \& Groll, S. (2005). Audience-tuning effects on memory: The role of shared reality. Journal of Personality \& Social Psychology, 89, 257-276. doi:10.1037/0022-3514.89.3.257

Echterhoff, G., Higgins, E. T., Kopietz, R., \& Groll, S. (2008). How communication goals determine when audience tuning biases memory. Journal of Experimental Psychology: General, 137, 3-21. doi:10.1037/0096-3445.137.1.3

Echterhoff, G., Higgins, E. T., \& Levine, J. M. (2009). Shared reality: Experiencing commonality with others' inner states about the world. Perspectives on Psychological Science, 4, 496-521.

Finlay, F., Hitch, G. J., \& Meudell, P. R. (2000). Mutual inhibition in collaborative recall: Evidence for a retrieval-based account. Journal of Experimental Psychology: Learning, Memory, \& Cognition, 26, 1556-1567. doi:10.1037/0278-7393.26.6.1556

French, L., Garry, M., \& Mori, K. (2008). You say tomato? Collaborative remembering leads to more false memories for intimate couples than for strangers. Memory, 16, 262-273. doi:10.1080/ 09658210701801491

Gabbert, F., Memon, A., Allan, K., \& Wright, D. B. (2004). Say it to my face: Examining the effects of socially encountered misinformation. Legal \& Criminological Psychology, 9, 215-227. doi:10.1348/ 1355325041719428

Gabbert, F., Memon, A., \& Wright, D. B. (2006). Memory conformity: Disentangling the steps toward influence during a discussion. Psychonomic Bulletin \& Review, 13, 480-485.
Gabbert, F., Memon, A., \& Wright, D. B. (2007). I saw it for longer than you: The relationship between perceived encoding duration and memory conformity. Acta Psychologica, 124, 319-331. doi:10.1016/j .actpsy.2006.03.009

GatherCole, S. E. (2008). Working memory. In J. H. Byrne (Series Ed.) \& H. L. Roediger III (Vol. Ed.), Learning and memory: A comprehensive reference. Vol. 2: Cognitive psychology of memory (pp. 33-51). Amsterdam: Elsevier.

Graf, P., \& Schacter, D. L. (1985). Implicit and explicit memory for new associations in normal and amnesic subjects. Journal of Experimental Psychology: Learning, Memory, \& Cognition, 11, 501-518. doi:10.1037/0278-7393.11.3.501

Halbwachs, M. (1980). The collective memory. (F. J. Ditter, Jr. \& V. Y. Ditter, Trans.). New York: Harper Row. (Original work published 1950)

Harris, C. B., Paterson, H. M., \& Kemp, R. I. (2008). Collaborative recall and collective memory: What happens when we remember together? Memory, 16, 213-230. doi:10.1080/09658210701811862

Higgins, E. T., \& RHoles, W. S. (1978). "Saying is believing": Effects of message modification on memory and liking for the person described. Journal of Experimental Social Psychology, 14, 363-378. doi:10.1016/0022-1031(78)90032-x

Hinsz, V. B., Tindale, R. S., \& Vollrath, D. A. (1997). The emerging conceptualization of groups as information processors. Psychological Bulletin, 121, 43-64. doi:10.1037/0033-2909.121.1.43

Hirst, W., \& MANiER, D. (2008). Towards a psychology of collective memory. Memory, 16, 183-200. doi:10.1080/09658210701811912

Jenkins, J. J. (1979). Four points to remember: A tetrahedral model of memory experiments. In L. S. Cermak \& F. I. M. Craik (Eds.), Levels of processing in human memory (pp. 429-446). Hillsdale, NJ: Erlbaum.

Johansson, N. O., Andersson, J., \& RönnberG, J. (2005). Compensating strategies in collaborative remembering in very old couples. Scandinavian Journal of Psychology, 46, 349-359. doi:10.1111/j.1467 $-9450.2005 .00465 . \mathrm{x}$

KaraU, S. J., \& Williams, K. D. (1993). Social loafing: A meta-analytic review and theoretical integration. Journal of Personality \& Social Psychology, 65, 681-706. doi:10.1037/0022-3514.65.4.681

Krause, U.-M., Stark, R., \& MandL, H. (2009). The effects of cooperative learning and feedback on e-learning in statistics. Learning \& Instruction, 19, 158-170. doi:10.1016/j.learninstruc.2008.03.003

Kwon, S. Y., \& Cifuentes, L. (2007). Using computers to individuallygenerate vs. collaboratively-generate concept maps. Educational Technology \& Society, 10, 269-280.

Kwon, S. Y., \& Cifuentes, L. (2009). The comparative effect of individually-constructed vs. collaboratively-constructed computer-based concept maps. Computers \& Education, 52, 365-375. doi:10.1016/j .compedu.2008.09.012

Laughlin, P. R., \& Sweeney, J. D. (1977). Individual-to-group and group-to-individual transfer in problem solving. Journal of Experimental Psychology: Human Learning \& Memory, 3, 246-254. doi:10.1037/0278-7393.3.2.246

Liang, D. W., Moreland, R., \& Argote, L. (1995). Group versus individual training and group performance: The mediating role of transactive memory. Personality \& Social Psychology Bulletin, 21, 384-393. doi:10.1177/0146167295214009

MÄNTYLÄ, T., \& Nilsson, L.-G. (1983). Are my cues better than your cues? Uniqueness and reconstruction as prerequisites for optimal recall of verbal materials. Scandinavian Journal of Psychology, 24, 303312. doi:10.1111/j.1467-9450.1983.tb00504.x

MARSH, E. J. (2007). Retelling is not the same as recalling: Implications for memory. Current Directions in Psychological Science, 16, 16-20. doi:10.1111/j.1467-8721.2007.00467.x

Marsh, E. J., \& TVERSKy, B. (2004). Spinning the stories of our lives. Applied Cognitive Psychology, 18, 491-503. doi:10.1002/acp.1001

MeAde, M. L., \& Roediger, H. L., III (2002). Explorations in the social contagion of memory. Memory \& Cognition, 30, 995-1009.

Merckelbach, H., Van Roermund, H., \& Candel, I. (2007). Effects of collaborative recall: Denying true information is as powerful as suggesting misinformation. Psychology, Crime \& Law, 13, 573-581. doi:10.1080/10683160601160679

Meudell, P. R., Hitch, G. J., \& Kirby, P. (1992). Are two heads better than one? Experimental investigations of the social facilitation 
of memory. Applied Cognitive Psychology, 6, 525-543. doi:10.1002/ acp. 2350060606

Mulligan, N. W., \& Lozito, J. P. (2006). An asymmetry between memory encoding and retrieval: Revelation, generation, and transfer-appropriate processing. Psychological Science, 17, 7-11. doi:10.1111/j.1467-9280.2005.01657.x

Nistad, B. A., \& Stroebe, W. (2006). How the group affects the mind: A cognitive model of idea generation in groups. Personality \& Social Psychology Review, 10, 186-213. doi:10.1207/s15327957pspr1003_1

Pasupathi, M., LuCAs, S., \& CoOmbs, A. (2002). Conversational functions of autobiographical remembering: Long-married couples talk about conflicts and pleasant topics. Discourse Processes, 34, 163-192. doi:10.1207/s15326950dp3402_3

Pasupathi, M., Stallworth, L. M., \& Murdoch, K. (1998). How what we tell becomes what we know: Listener effects on speakers' long-term memory for events. Discourse Processes, 26, 1-25.

PAulus, P. (2000). Groups, teams, and creativity: The creative potential of idea-generating groups. Applied Psychology, 49, 237-262. doi:10.1111/1464-0597.00013

PeltokoRpi, V. (2008). Transactive memory systems. Review of General Psychology, 12, 378-394. doi:10.1037/1089-2680.12.4.378

RAJARAM, S., \& BARBER, S. J. (2008). Retrieval processes in memory. In J. H. Byrne (Series Ed.) \& H. L. Roediger III (Vol. Ed.), Learning and memory: A comprehensive reference. Vol. 2: Cognitive psychology of memory (pp. 261-283). Amsterdam: Elsevier.

Rajaram, S., \& Pereira-Pasarin, L. P. (2007). Collaboration can improve individual recognition memory: Evidence from immediate and delayed tests. Psychonomic Bulletin \& Review, 14, 95-100.

REYSEN, M. B. (2007). The effects of social pressure on false memories. Memory \& Cognition, 35, 59-65.

Roediger, H. L., III, Meade, M. L., \& Bergman, E. T. (2001). Social contagion of memory. Psychonomic Bulletin \& Review, 8, 365-371.

Ross, M., Spencer, S. J., Blatz, C. W., \& Restorick, E. (2008). Collaboration reduces the frequency of false memories in older and younger adults. Psychology \& Aging, 23, 85-92. doi:10.1037/0882 $-7974.23 .1 .85$

Ross, M., Spencer, S. J., Linardatos, L., Lam, K. C. H., \& PeRUNOVIC, M. (2004). Going shopping and identifying landmarks: Does collaboration improve older people's memory? Applied Cognitive Psychology, 18, 683-696. doi:10.1002/acp.1023

SlameCKA, N. J. (1969). Testing for associative storage in multitrial free recall. Journal of Experimental Psychology, 81, 557-560. doi:10.1037/ h0027909
Thorley, C., \& Dewhurst, S. A. (2007). Collaborative false recall in the DRM procedure: Effects of group size and group pressure. European Journal of Cognitive Psychology, 19, 867-881. doi: $10.1080 / 09541440600872068$

Tulving, E. (1983). Elements of episodic memory. New York: Oxford University Press.

Tulving, E., \& Osler, S. (1968). Effectiveness of retrieval cues in memory for words. Journal of Experimental Psychology, 77, 593601. doi:10.1037/h0026069

WeGNer, D. M. (1987). Transactive memory: A contemporary analysis of the group mind. In B. Mullen \& G. R. Goethals (Eds.), Theories of group behavior (pp. 185-208). New York: Springer.

Wegner, D. M., ERbER, R., \& RAYMOND, P. (1991). Transactive memory in close relationships. Journal of Personality \& Social Psychology, 61, 923-929. doi:10.1037/0022-3514.61.6.923

Weldon, M. S. (2001). Remembering as a social process. In D. L. Medin (Ed.), The psychology of learning and motivation: Advances in research and theory (Vol. 40, pp. 67-120). San Diego: Academic Press.

Weldon, M. S., \& Bellinger, K. D. (1997). Collective memory: Collaborative and individual processes in remembering. Journal of Experimental Psychology: Learning, Memory, \& Cognition, 23, 1160 1175. doi:10.1037/0278-7393.23.5.1160

Weldon, M. S., Blair, C., \& Huebsch, P. D. (2000). Group remembering: Does social loafing underlie collaborative inhibition? Journal of Experimental Psychology: Learning, Memory, \& Cognition, 26, 1568-1577. doi:10.1037/0278-7393.26.6.1568

Wertsch, J. V. (2002). Voices of collective remembering. Cambridge: Cambridge University Press.

WiLson, M. (1988). MRC Psycholinguistic Database: Machine-usable dictionary, version 2.00. Behavior Research Methods, Instruments, \& Computers, 20, 6-10.

YUKER, H. E. (1955). Group atmosphere and memory. Journal of Abnormal \& Social Psychology, 51, 17-23. doi:10.1037/h0046464

\section{NOTE}

1. Participants from the encode dyadically-recall dyadically with a different partner condition were not included in these analyses, because the dyad at retrieval was only partially the same as the dyad at encoding.

(Manuscript received June 6, 2009; revision accepted for publication October 22, 2009.) 